\title{
Increasing Tip60 HAT Levels Rescues Axonal Transport Defects and Associated Behavioral Phenotypes in a Drosophila Alzheimer's Disease Model
}

\author{
Ashley A. Johnson, ${ }^{\star}$ Jessica Sarthi, ${ }^{\star}$ Sheila K. Pirooznia, ${ }^{\star *}$ William Reube, ${ }^{\star \star}$ and Felice Elefant \\ Department of Biology, Drexel University, Philadelphia, Pennsylvania 19104
}

\begin{abstract}
Axonal transport defects and axonopathy are prominent in early preclinical stages of Alzheimer's disease (AD), often preceding known disease-related pathology by over a year. As epigenetic transcriptional regulatory mechanisms, such as histone acetylation, are critical for neurogenesis, it is postulated that their misregulation might be linked to early pathophysiological mechanisms that contribute to AD. The histone acetyltransferase (HAT) Tip60 epigenetically regulates genes enriched for neuronal functions and is implicated in AD via its formation of a transcriptional regulatory complex with the amyloid precursor protein (APP) intracellular domain. Disruption of APP function is associated with axonal transport defects, raising the possibility that an epigenetic role for Tip60 might also be involved. Here, we examine whether Tip60 HAT activity functions in axonal transport using Drosophila CNS motor neurons as a well-characterized transport model. We show that reduction of Tip $60 \mathrm{HAT}$ activity in the nervous system causes axonopathy and transport defects associated with epigenetic misregulation of certain axonal transport-linked Tip60 target genes. Functional consequences of these defects are evidenced by reduced locomotion activity of the mutant Tip60 larvae, and these phenotypes can be partially rescued with certain histone deacetylase inhibitors. Finally, we demonstrate that Tip60 function in axonal transport is mediated by APP and that, remarkably, excess Tip60 exerts a neuroprotective role in APP-induced axonal transport and functional locomotion defects. Our observations highlight a novel functional interactive role between Tip60 HAT activity and APP in axonal transport and provide insight into the importance of specific HAT modulators for cognitive disorder treatment.
\end{abstract}

\section{Introduction}

Epigenetic mechanisms of gene control via histone acetylation regulate neuronal patterning and activity during neurogenesis that influence cognitive function (Carrozza et al., 2003; Wang et al., 2010). It remains unclear, however, which specific histone acetyltransferase (HAT) enzymes control these processes. The HAT Tip60 functions in chromatin-mediated processes, including gene regulation and apoptosis, with emerging roles in neuronal function (Sapountzi et al., 2006; Squatrito et al., 2006). We previously reported that Tip60 HAT activity is required for neuronal gene control (Lorbeck et al., 2011), regulation of synaptic plasticity (Sarthi and Elefant, 2011), sleep (Pirooznia et al., 2012a), and apoptosis in the Drosophila CNS (Pirooznia et al., 2012b). Accordingly, Tip60 is implicated in the neurodegenera-

\footnotetext{
Received Aug. 4, 2012; revised Jan. 16, 2013; accepted March 15, 2013.

Author contributions: A.A.J., J.S., W.R., and F.E. designed research; A.A.J., J.S., and W.R. performed research; J.S. and S.K.P. contributed unpublished reagents/analytic tools; A.A.J., J.S., S.K.P., W.R., and F.E. analyzed data; A.A.J. and F.E. wrote the paper.

This work was supported by National Institutes of Health Grant R01HD057939 to F.E. We thank Dr. Kendal Broadie for generously providing the UAS-sty-eGFP fly lines and the Cell Imaging Center at Drexel University for their imaging facilities.

The authors declare no competing financial interests.

${ }^{*}$ A.A.J. and J.S. contributed equally to this work as first authors.

* S.K.P. and W.R. contributed equally to this work as second authors.

Correspondence should be addressed to Dr. Felice Elefant, Department of Biology, Drexel University, 3141 Chestnut Street, Philadelphia, PA 19104. E-mail: fe22@drexel.edu.

DOI:10.1523/JNEUROSCI.3739-12.2013

Copyright $\odot 2013$ the authors $\quad 0270-6474 / 13 / 337535-13 \$ 15.00 / 0$
}

tive disorder Alzheimer's disease (AD) via its formation of a transcriptional regulatory complex with the $\mathrm{AD}$ associated amyloid precursor protein (APP) intracellular domain (AICD) (Cao and Sudhof, 2001; Slomnicki and Lésnizk, 2008). Aberrant epigenetic regulation of certain genes has been linked to $\mathrm{AD}$, suggesting that Tip60-dependent mechanisms may underlie the disorder (Baek et al., 2002). In support of this concept, we reported that Tip60 HAT activity mediates APP-induced neuronal cell death in the CNS of an AD Drosophila model via misregulation of apoptosislinked genes (Pirooznia et al., 2012b).

Accumulating evidence suggests that cognitive impairment in $\mathrm{AD}$ and nervous system processes affecting neural connections and activity are disrupted during early stages of the disease (Trinchese et al., 2008). Axonopathy and axonal transport defects are commonly observed in early preclinical stages of $\mathrm{AD}$, often preceding amyloid deposition and neuronal cell death by over a year in mouse AD models (Stokin et al., 2005). Accumulation of axonal transport defects is thought to contribute to neurodegenerative disorder progression by triggering apoptotic pathways that lead to loss of neural activity (Roy et al., 2005; Duncan and Goldstein, 2006; Stokin and Goldstein, 2006). Early epigenetic transcriptional regulatory mechanisms involving histone acetylation are critical in neurogenesis and; as such, their misregulation may contribute to early pathophysiological mechanisms underlying progressive cognitive disorders (Levenson et al., 2004; Peleg et al., 2010; Sweatt). Disruption of APP function has been linked to axonal transport defects (Torroja et al., 1999; Gunawar- 
dena and Goldstein, 2001), raising the possibility that an epigenetic role for Tip60 might also be involved.

Here, we investigate whether Tip60 plays a role in axonal transport using Drosophila CNS motor neurons as a wellcharacterized transport model (Budnik and Ruiz-Canada, 2006). We show that loss of Tip60 HAT activity in the nervous system causes axonopathy associated with epigenetic misregulation of axonal transport-linked Tip60 direct target genes. Functional consequences of these defects are evidenced by reduced locomotor activity of mutant Tip60 larvae, and these phenotypes can be rescued with certain histone deacetylase (HDAC) inhibitors. We show that Tip60 function in axonal transport is mediated by APP and that excess Tip60 exerts a neuroprotective role in APPinduced axonal transport defects. Our observations highlight an interactive role between Tip60 and APP in axonal transport and provide insight into understanding epigenetic HAT-based mechanisms underlying early pathophysiology of AD.

\section{Materials and Methods}

Fly stains and crosses. All fly lines used were raised under standard conditions at $22^{\circ} \mathrm{C}$ on standard yeasted Drosophila media (Applied Scientific Jazz Mix Drosophila Food, Thermo Fisher Scientific). The pan-neuronal driver elav ${ }^{\mathrm{c} 155}$ and the motor neuron-specific driver D42 were obtained from Bloomington Stock Center. Independent transgenic fly line dTip60 ${ }^{\mathrm{WT}}$ (line C), dTip60 ${ }^{\mathrm{E} 431 \mathrm{Q}}$ (independent lines A and B), and dTip60 ${ }^{\mathrm{RNAi}}$ (line A) were created as previously described (Zhu et al., 2007; Lorbeck et al., 2011). Rescue lines dTip60 ${ }^{\text {Rescue }} \mathrm{B}$ and dTip $60{ }^{\text {Rescue }} \mathrm{C}$ were generated and fully characterized as previously reported (Lorbeck et al., 2011). dTip60 Rescue $\mathrm{B}$ is the $\mathrm{dTip} 60^{\mathrm{E} 431 \mathrm{Q}} \mathrm{B}$ fly line containing an extra copy of dTip $60{ }^{\mathrm{WT}}$ crossed, and dTip $60{ }^{\text {Rescue }} \mathrm{C}$ is the dTip60 ${ }^{\mathrm{E} 431 \mathrm{Q}} \mathrm{A}$ fly line containing an extra copy of dTip60 ${ }^{\mathrm{WT}}$. APP; dTip60 ${ }^{\mathrm{E} 431 \mathrm{Q}}$ (independent lines A and B), APP dCT; dTip60 ${ }^{\mathrm{E} 431 \mathrm{Q}}$ (independent lines $\mathrm{A}$ and B); APP;dTip60WT (line C), and APP dCT; dTip $60^{\text {WT }}$ (line C) were created as previously described by standard genetic crosses (Pirooznia et al., 2012b). The fly line containing elavGAL4-syteGFP was a generous gift from Dr. Kendal Broadie (Vanderbilt University, Nashville, TN). All experimental crosses were performed at normal physiological temperature of $25^{\circ} \mathrm{C}$.

Larval motor function assays. Wandering third instar larvae were used for all larval motor function assays. The following three larval motor function assays were performed as previously described (Mudher et al., 2004) to quantify locomotor dysfunction. Before the start of the test, the larvae are placed on a Petri dish containing $1 \%$ agarose and allowed to equilibrate for $5 \mathrm{~min}$. In the contraction assay, the number of full body peristaltic contractions performed in $30 \mathrm{~s}$ was recorded. In the righting assay, larvae were turned ventral side up and the time taken to return to the crawling position was recorded. In the line crossing assay, the number of lines crossed by the head of a larva in $30 \mathrm{~s}$ on a grid $0.4 \mathrm{~cm} \times 0.4 \mathrm{~cm}$ was recorded. At least 30 larvae were tested in each locomotor assay three times, and unpaired Student's $t$ test was used for statistical analysis.

Axonal transport analysis. Wandering third instar larvae were dissected in HL-3 saline, pH 7.2, and pinned out on Sylgard dishes by cutting along the length of the dorsal midline and removing the digestive track and trachea to expose the CNS. Fillet preparations were fixed in 3.5\% paraformaldehyde for $30 \mathrm{~min}$, followed by 3 washes in PBS (10 min each), then mounted onto slides with Vectashield antifade mounting media (Vector Laboratories).

Imaging of axonal transport analysis. Quantitative analysis of larval motor neurons was performed as follows. Five different nerves were selected from the region immediately posterior to the brain. Larval motor neurons were imaged on an Olympus FV1000 laser scanning confocal microscope. All images were captured using same constant confocal gain settings. Images were acquired as a Z-stack and then rendered as a maximum projection. The nerves were analyzed by counting synaptotagmin accumulations with a diameter of at least $0.7 \mu \mathrm{m}$ along a segment of at least $50 \mu \mathrm{m}$. In the analysis, each genotype is represented by $10-15$ larval fillet preparations. For comparison between genotypes, all samples were processed simultaneously and imaged using identical microscopic acquisitions parameters. Unpaired Student's $t$ test was used for statistical analysis.

Immunohistochemistry and imaging analysis. Third instar larvae were filleted in HL-3 saline, $\mathrm{pH} 7.2$ and pinned out on Sylgard dishes with guts removed. Fillet preparations were fixed in 3.5\% paraformaldehyde for 30 min, followed by 3 washes in PBS and one with $1 \times$ PBS with $0.1 \%$ Triton $\mathrm{X}-100$. The larvae were then incubated overnight in primary antibody at $4^{\circ} \mathrm{C}$. The primary mouse monoclonal anti-acetylated tubulin $(1: 1000)$ antibody was obtained from Sigma-Aldrich. After 6 washes in PBS-T $(1 \times$ PBS $+0.1 \%$ Triton X-100), fillets were incubated in $2^{\circ}$ antibody for $1 \mathrm{~h}$, washed twice in $1 \times$ PBS-T, once in $1 \times$ PBS, then mounted onto slides in Vectashield antifade mounting media (Vector Laboratories). Confocal microscopy was performed using an Olympus microscope with fluoview software. All images were captured using same constant confocal gain settings. Images were acquired as a Z-stack and then rendered as a maximum projection. In the analysis, each genotype is represented by $10-15$ larval fillet preparations. For comparison between genotypes, all samples were processed simultaneously and imaged using identical microscopic acquisitions parameters. Quantitative analysis of fluorescence intensities was done using FV1000 software. All images were corrected for background before intensity measurements were performed. Unpaired Student's $t$ test was used for statistical analysis.

Real-time PCR analysis. Total RNA was isolated from wandering third instar dTip $60{ }^{\mathrm{E} 431 \mathrm{Q}} \mathrm{B}$ and wild-type larvae using the RNeasy Plus Mini Kit (QIAGEN). cDNA was prepared using the SuperScript II reverse transcriptase kit (Invitrogen) according to the manufacturer's instructions with $1 \mu \mathrm{g}$ total RNA and $0.2 \mu \mathrm{g} / \mathrm{ml}$ random hexamer primers (Roche Applied Science). PCRs were performed in a $20 \mu \mathrm{l}$ reaction volume containing cDNA, $1 \times$ Power SYBR Green PCR Master Mix (Applied Biosystems), and $10 \mu \mathrm{M}$ both forward and reverse primers (primer pairs available upon request). PCR was performed using an ABI 7500 RealTime PCR system (Applied Biosystems) following the manufacturer's instructions. Fold change in mRNA expression was determined by the $\Delta \Delta$ Ct method (Livak and Schmittgen, 2001).

Chromatin immunoprecipitation (ChIP) and qPCR analysis. Chromatin from third instar larval heads was prepared as follows. Approximately 300 third instar larval heads for each genotype were homogenized first with a pestle and then using a potter homogenizer ( 5 strokes) containing homogenization buffer (60 mM KCl, $15 \mathrm{~mm} \mathrm{NaCl}, 4 \mathrm{~mm} \mathrm{MgCl}_{2}, 15 \mathrm{~mm}$ HEPES, pH 7.6, 0.5\% Triton X-100, 0.5 mm DTT, protease and phosphatase inhibitors mixture; Roche). Cross-linking was performed for $15 \mathrm{~min}$ by adding formaldehyde to a final concentration of $1.8 \%$. Cross-linking reaction was stopped by adding glycine solution (final concentration of $225 \mathrm{~mm}$ ) followed by incubation on ice for $5 \mathrm{~min}$. Fixed tissue homogenate was then washed $3 \times$ homogenization buffer and $1 \times$ in lysis buffer (140 mm NaCl, 15 mm HEPES, pH 7.6, 1 mm EDTA, 0.5 mм EGTA, 1\% Triton X-100, $0.5 \mathrm{~mm}$ DTT, $0.1 \%$ sodium deoxycholate, protease and phosphatase inhibitors [Roche], $1 \mathrm{~mm}$ PMSF, and $10 \mathrm{~mm}$ sodium butyrate). Next, lysis buffer containing SDS (0.1\%) and sodium lauryl sarcosine $(0.5 \%)$ was added to the homogenate and incubated for $10 \mathrm{~min}$ at $4^{\circ} \mathrm{C}$. After incubation, the cells were disrupted by 8 pulses for $30 \mathrm{~s}$ each on ice using a tip sonicator (Fisher Scientific Sonic Dismembrator 50 Ultrasonic Homogenizer), yielding sheared chromatin fragments ranging from 200 to $500 \mathrm{bp}$. The sheared chromatin was then incubated at $4^{\circ} \mathrm{C}$ for $10 \mathrm{~min}$ and centrifuged twice, yielding the final chromatin extract for ChIP.

Chromatin precipitation assays were performed using ChIP-IT Express Kit (Active Motif), following the manufacturer's instructions. Briefly, ChIP was performed with $20 \mu \mathrm{g}$ sheared chromatin for H4 panacetylated antibody (1:150) (AbD Serotec) and $30 \mu \mathrm{g}$ sheared chromatin for Tip60 antibody $(2 \mu \mathrm{g})$ (Abcam). A mock reaction containing all reagents, except the antibody, was performed simultaneously for each sample as a control. The chromatin was immunoprecipitated using the ChIP IT Express kit (Active Motif) exactly following the manufacturer's specifications. The eluted material from the immunoprecipitation was then purified using QIAquick PCR purification kit (QIAGEN) and was directly used for real-time PCR.

PCRs were performed in triplicate a $20 \mu \mathrm{l}$ reaction volume containing $10 \mu$ l of SYBR Green PCR master mix (Applied Biosystems), $1 \mu$ l of DNA 
template, and $0.5 \mu \mathrm{M}$ each of forward and reverse primer for each gene sequence (primer pairs available upon request). Quantitative PCR was performed on an ABI 7500 Real Time PCR System (Applied Biosystems) according to the manufacturer's instructions. For each primer set, fold enrichment was calculated using the slope of a standard curve according to the manufacturer's instructions in the ChIP-IT Express kit. Briefly, a standard curve was generated from serial 10-fold dilutions of the Input DNA. The $C_{t}$ values were used to estimate DNA quantity of the ChIP and no antibody mock control samples. Fold enrichment was calculated as a ratio of the DNA quantity in the ChIP and no antibody mock control.

Drug treatment. Dried potato food (Carolina Biological Supply Company) was made in $3 \mathrm{ml}$ of water with the desired final concentration of the following HDAC inhibitors (HDACi): ms-275 (LC Laboratories), sodium butyrate (LC Laboratories), SAHA (LC Laboratories), Givinostat (Selleck Chemicals), valproic acid (Selleck Chemicals), Romidepsin (Selleck Chemicals), Mocetinostat (Selleck Chemicals), or no drug (all drug concentrations used available upon request). Mated flies for each genotype were allowed to lay eggs on drug-treated food until the third instar stage in which they were used for the relevant experiments. Doseresponse curve experiments were performed on wild-type larvae using different titrations of each drug and the highest concentration of drug that caused no observable locomotion defects in wild-type larvae was selected for use.

\section{Results}

\section{Loss of dTip60 HAT function in the CNS impairs larval locomotor function}

We previously demonstrated that dTip60 is robustly expressed in the embryonic ventral midline cells destined to become the motor neurons (Lorbeck et al., 2011). To test whether Tip60 HAT activity is required for motor neuron function, the GAL4targeted system was used to modulate dTip60 HAT activity levels in the nervous system, and a series of functional locomotion assays was performed. Misregulation of dTip60 was achieved for all experiments by using the GAL4/UAS targeted gene expression system with flies carrying UAS-responsive transgenes for wildtype dTip60 (dTip60 ${ }^{\text {WT }}$ ), dTip60 ${ }^{\text {RNAi }}$ (Zhu et al., 2007; Sarthi and Elefant, 2011; Pirooznia et al., 2012b), or a dominantnegative HAT-defective version of $\mathrm{dTip} 60^{\mathrm{E} 431 \mathrm{Q}} \mathrm{using}$ independent fly lines dTip $60{ }^{\mathrm{E} 431 \mathrm{Q}} \mathrm{A}$ and $\mathrm{dTip} 60{ }^{\mathrm{E} 431 \mathrm{Q}} \mathrm{B}$ that express low and high levels of the dTip $60^{\mathrm{E} 431 \mathrm{Q}}$ transgene, respectively (Lorbeck et al., 2011). Fly lines dTip60 ${ }^{\mathrm{RNAi}}$, dTip60 ${ }^{\mathrm{E} 431 \mathrm{Q}}$, dTip60 ${ }^{\mathrm{WT}}$, and $w^{1118}$ control flies were each crossed to the presynaptic pan-neuronal GAL4 driver elav ${ }^{\mathrm{C} 155}$. Initial qualitative observations of motor function in wandering third instar larvae showed that flies with reduced dTip60 HAT function exhibited slow, sluggish, and disjointed movements. The most severely affected larvae exhibited posterior paralysis and a distinctive tail flipping phenotype, which causes the larvae to rhythmically flip their tails upward while crawling. This phenotype is reminiscent of those described for mutants with known defects in vesicle transport machinery, such as Kinesin heavy chain (Hurd and Saxton, 1996) and Kinesin light chain (Gindhart et al., 1998) mutants.

To quantify such locomotor dysfunction, a series of three larval motor function assays were performed as previously described (Mudher et al., 2004). Contraction capability, speed of locomotion, and ability to perform a complex motor task, such as righting, provide direct, quantifiable evidence of motor neuron function. In the contraction assay, the number of full body peristaltic contractions performed in $30 \mathrm{~s}$ was recorded. In the righting assay, larvae were turned ventral side up and the time taken to return to the crawling position was recorded. In the line crossing assay, the number of lines crossed by the head of a larva in $30 \mathrm{~s}$ on a grid $0.4 \mathrm{~cm} \times 0.4 \mathrm{~cm}$ was recorded. Our results show that induction of dTip $60^{\mathrm{RNAi}}$ and dTip $60^{\mathrm{E} 431 \mathrm{Q}}$ in the nervous system using the GAL4 driver elav ${ }^{\mathrm{C} 155}$ resulted in larvae that were significantly less mobile and crossed proximately half as many grid lines compared with control wild-type larvae from a $w^{1118}$ x elav-GAL4 cross (Fig. 1A), performed significantly fewer full body peristaltic contractions (Fig. $1 B$ ) and took on average three times longer to return to the crawling position (Fig. $1 C)$. Similar results were obtained when dTip $60^{\mathrm{RNAi}}$ and dTip $60^{\mathrm{E} 431 \mathrm{Q}}$ were induced using the motor neuron-specific GAL4 driver D42 (Fig. 1D), confirming that these locomotion defects were specifically the result of a loss of motor neuron function. Induction of dTip $60^{\mathrm{WT}}$ had no effect on locomotor ability (Fig. $1 A-C$ ). Moreover, dTip $60^{\mathrm{RNAi}} \mathrm{A}$ and dTip $60{ }^{\mathrm{E} 431 \mathrm{Q}}$ locomotor defects were fully rescued by an additional copy of dTip60 ${ }^{\text {WT }}$ (Fig. 1A-C), indicating that the phenotypic effects we observe are specifically caused by disruption of Tip60 HAT function. Together, these results suggest that loss of dTip60 function in the nervous system, and specifically in the motor neurons, results in locomotor dysfunction in the fly.

\section{Nervous system-specific disruption of Tip60 HAT activity causes vesicle accumulations in motor axons extending from the CNS}

The tail flipping phenotype and locomotion defects that result from disruption of Tip60 HAT activity are reminiscent of nervous system defects linked to mutations in genes required for axonal vesicle transport machinery. These findings prompted us to assess whether axonal transport was perturbed by misregulation of Tip60 HAT activity by using double transgenic flies coexpressing a synaptotagmin-e-GFP fusion protein (syt-eGFP) along with either dTip $60^{\mathrm{E} 431 \mathrm{Q}}$ or dTip $60^{\mathrm{WT}}$ under the control of the pan-neuronal elav-GAL4 driver. Synaptotagmin is an integral synaptic vesicle protein; thus, the syt-eGFP fusion protein serves as a well-characterized marker to assess synaptic vesicle transport along the axons in the fly (Zhang et al., 2002). As axonal transport defects often manifest as axonal swellings or spheroids corresponding to axonal enlargements and aberrant accumulations of axonal cargos (Stokin et al., 2005), such axonopathy can be readily identified and quantitated using the syt-eGFP fusion protein. Third instar wandering larvae were dissected to expose motor axons extending from the CNS, and confocal imaging was used to assess the distribution of GFP-tagged axonal vesicles. In control elav-GAL4-syt-eGFP larvae, GFP-tagged vesicles were evenly distributed through the length of the motor neuron (Fig. 2A), including the main intersegmental regions, the second-order nerve branches, and the neuromuscular junction where they were localized normally into boutons. In contrast, larvae expressing dTip $60^{\mathrm{E} 431 \mathrm{Q}}$ revealed the formation of aggregations of GFPtagged vesicles (Fig. $2 B, C$ ) thought to reflect defective passage of transport vesicles in peripheral axons (Gunawardena and Goldstein, 2001). As the diameter of an axon is $\sim 0.5 \mu \mathrm{m}$, abnormal axonal aggregations thought to disrupt axonal transport are defined as those $>0.7 \mu \mathrm{m}$ per $50 \mu \mathrm{m}$ of axonal length (Rusu et al., 2007). Quantification revealed a significantly larger number of these aggregates in flies expressing either dTip $600^{\mathrm{E} 431 \mathrm{Q}}$ construct (Fig. 2G) compared with control, dTip60 ${ }^{\mathrm{WT}}$, and dTip60 ${ }^{\text {Rescue }}$ flies where virtually no accumulations were observed (Fig. $2 A, D, E, F)$. Together, these results demonstrate that dTip60 HAT activity is required for synaptic vesicle transport along the motor axons in the fly. 
A
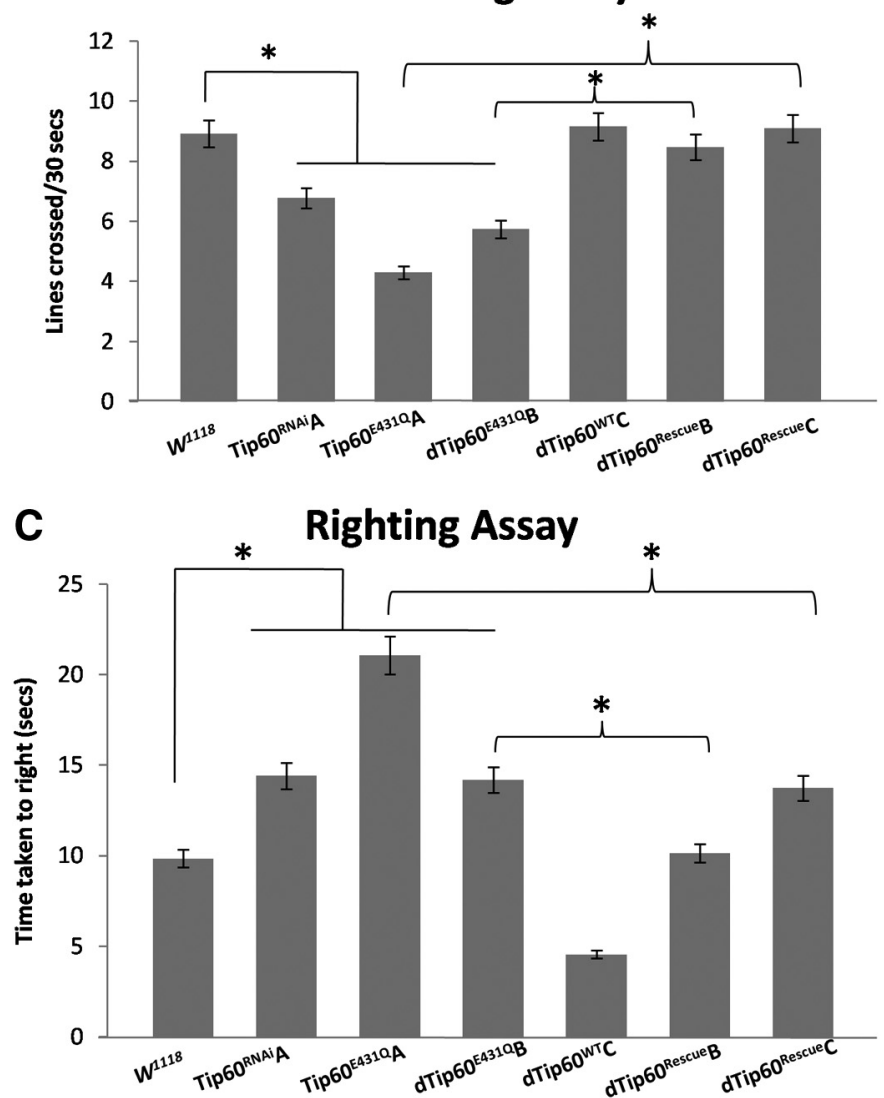

B

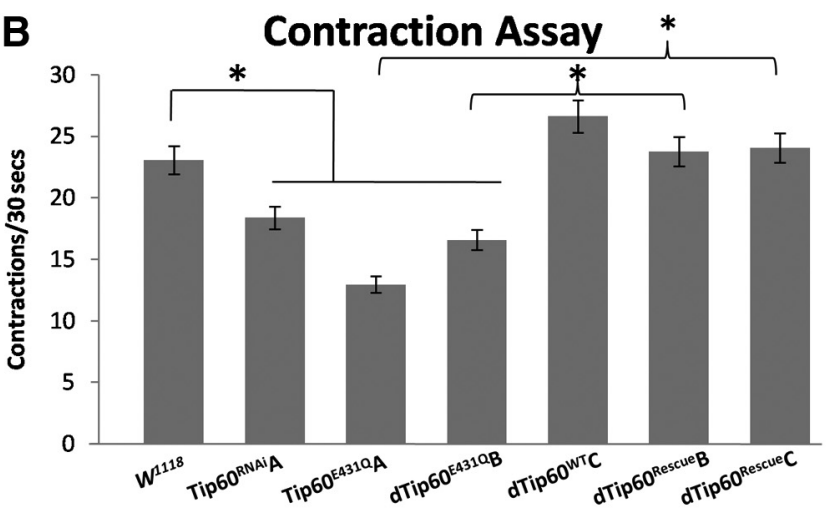

D Line-Crossing Assay



Figure 1. Reduction of dTip60 HAT activity in the CNS impairs larval locomotor function. Histograms representing the results of a series of locomotion assays conducted on $w^{1118}$ control third instar larvae or third instar larvae expressing each of the different GAL4 responsive transgenes under the control of the elav ${ }^{C 155}$ pan-neuronal GAL4 driver for the following assays: $A$, Line crossing assay. $\boldsymbol{B}$, Contraction assay. C, Righting assay. In all assays, larvae expressing dTip60 ${ }^{\mathrm{E} 4310}$ or dTip $60^{\mathrm{RNAi}}$ took significantly longer to complete the locomotor tasks and showed a significant loss of locomotor function compared with control and dTip60 ${ }^{\text {WT }}$ larvae. The defects observed in the dTip60 ${ }^{\text {E4310 }}$ larvae were rescued with an additional copy of Tip60 ${ }^{\text {WT }}$ (dTip60 Rescue independent lines

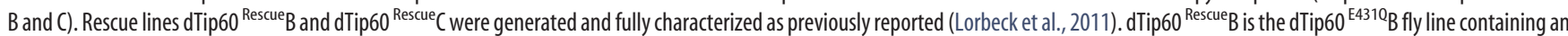
extra copy of dTip $60^{\mathrm{WT}}$ crossed, and dTip60 ${ }^{\text {Rescue }} \mathrm{C}$ is the $\mathrm{dTip} 60^{\mathrm{E} 4310} \mathrm{~A}$ fly line containing an extra copy of dTip60 ${ }^{\mathrm{WT}}$. D, Line crossing assay using the motor neuron-specific D42-GAL4 driver. Statistical significance for all experiments was calculated using an unpaired Student's $t$ test: ${ }^{*} p<0.05 . n=30$.

Tip60 HAT activity loss in the CNS does not impact tubulin acetylation status in the motor axons where vesicle stalling takes place

Acetylation of $\alpha$-tubulin within microtubules (MTs) is thought to regulate motor-dependent trafficking of cargo along neuronal axons (Hammond et al., 2008) by serving as a guidance cue for specific motors in which polarity has not yet fully been determined (Janke and Kneussel, 2010). In this regard, we previously reported that loss of Tip 60 within the fly neuromuscular junction results in reduction of acetylated MTs only at ends of axons specifically extending into the terminal synaptic boutons in conjunction with defects in bouton growth and development (Sarthi and Elefant, 2011). Thus, we asked whether MT acetylation was also reduced within the motor axons in the Tip60 HAT mutant flies as this might account for the vesicle accumulations we observe. To address this question, we performed immunohistochemical staining of the Drosophila motor neurons with anti-acetylated tubulin, which specifically recognizes $\alpha$-tubulin in the acetylated state. For these and all subsequent studies, we chose to use fly line $\mathrm{dTip} 60^{\mathrm{E} 431 \mathrm{Q}} \mathrm{B}$ as it expresses the most robust levels of dTip $60^{\mathrm{E} 431 \mathrm{Q}}$ of all fly lines tested and, accordingly, produces the most severe lethal phenotype and displays significantly depleted endogenous levels of acetylated H4 (Lorbeck et al., 2011). When larvae expressing dTip $60^{\mathrm{E} 431 \mathrm{Q}} \mathrm{B}$ were compared with $\mathrm{w}^{1118}$ con- trol larvae, the acetylated MT network appeared similar and, importantly, was present along the entire length of the motor axons where vesicle clogging is observed in dTip60 ${ }^{\mathrm{E} 431 \mathrm{Q}}$ larvae (Fig. $3 A-D)$. Quantitation of the mean fluorescence intensity of antiacetylated tubulin staining in these flies revealed that there was no significant difference between dTip $60^{\mathrm{E} 431 \mathrm{Q}}$ and control $\mathrm{w}^{1118}$ fly lines (Fig. $3 E$ ). These findings suggest that the synaptic vesicle clogging we observe within the motor axons in the dTip $60^{\mathrm{E} 431 \mathrm{Q}}$ fly line is not the result of reduced MT acetylation in these regions of the axons and may be mediated by another mechanism.

\section{Tip60 HAT activity in the CNS is required for the} transcriptional regulation of genes linked to axonal vesicle transport

Our previous analysis demonstrated that expression of dTip $60{ }^{\mathrm{E} 431 \mathrm{Q}}$ results in the misregulation of genes enriched for nervous system function (Lorbeck et al., 2011). As no changes were detected in the acetylation status of $\alpha$-tubulin within the motor axons of dTip $60^{\mathrm{E} 431 \mathrm{Q}}$ flies, we hypothesized that a potential mechanistic basis for the axonal transport phenotype caused by Tip60 HAT loss was the result of inappropriate expression of genes required for axonal transport. To test this hypothesis, we reanalyzed our previously published microarray gene expression data that compared changes in gene expression caused by ubiq- 
A

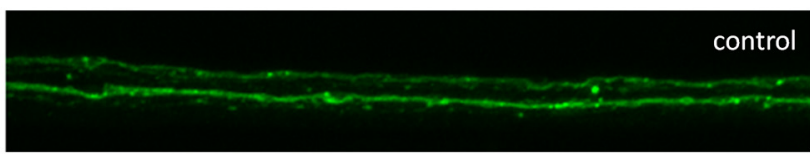

B

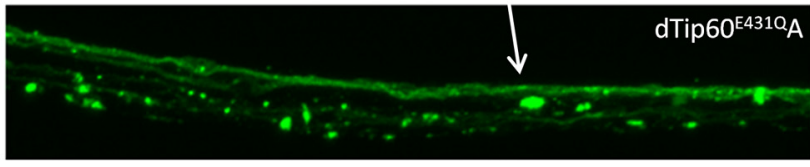

$\mathrm{C}$

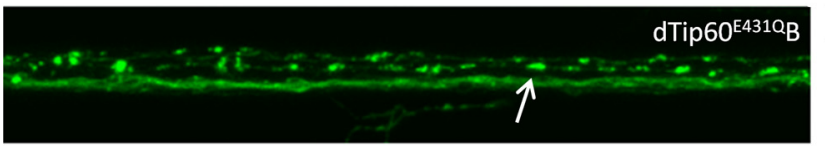

G
D
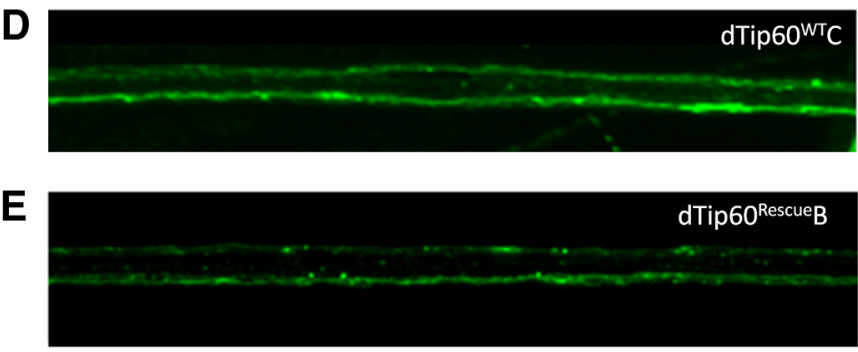

$\mathrm{F}$

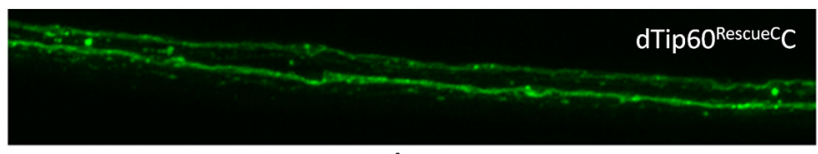

*

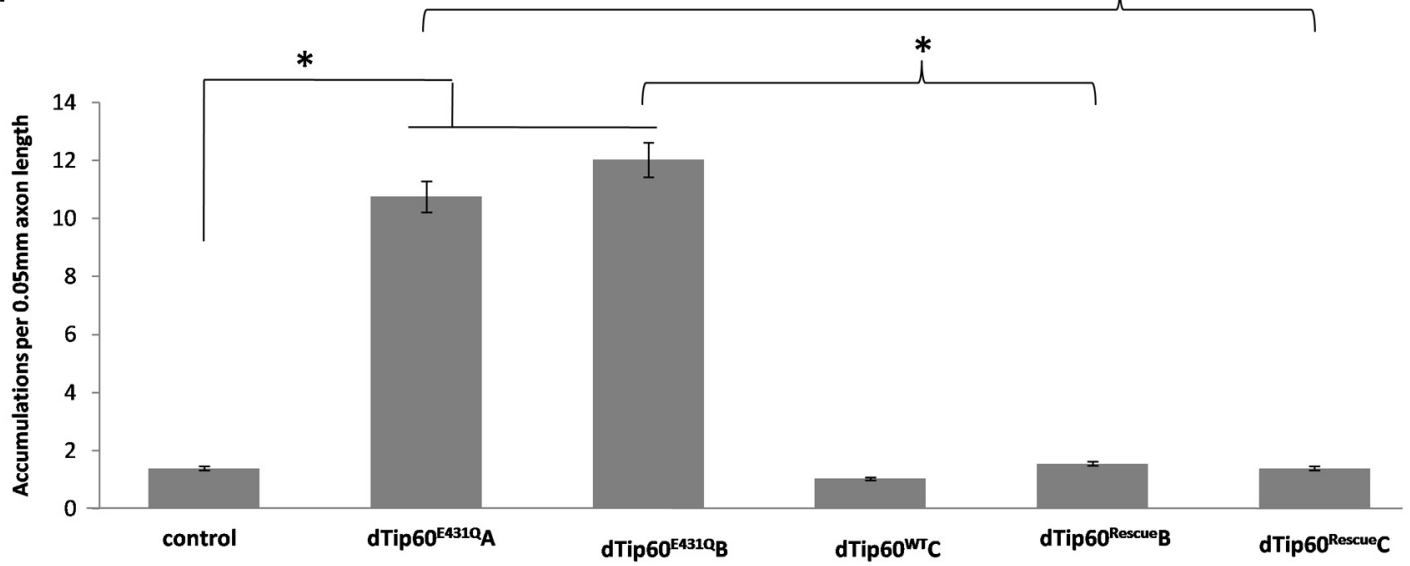

Figure 2. Nervous system-specific disruption of Tip60 HAT activity causes vesicle accumulations in motor axons extending from the CNS. Representative confocal images of motor axons directly posterior to brains in third instar larvae coexpressing a synaptotagmin-e-GFP fusion protein (syt-eGFP) along with either dTip6 ${ }^{\mathrm{E} 4310}$ or dTip60 ${ }^{\mathrm{WT}}$ under the control of the pan-neuronal elav-GAL4 driver at $25^{\circ}$ C. $A$, Control $w^{1118}$ larvae show no aggregations or accumulation. $B, C$, Larvae expressing dTip60 ${ }^{\mathrm{E} 4310}$ show significantly more aggregates than control. $\boldsymbol{D}$, Larvae expressing additional copies of wild-type Tip60 transgene (dTip60 WT) show no accumulations. $E, F$, Vesicle accumulation defects caused by dTip60 HAT loss are rescued by an additional copy of dTip60 ${ }^{\text {WT }}$ (dTip60 Rescue independent lines $B$ and C). Line arrows indicate synaptotagmin-positive vesicle aggregations. $G$, Histogram represents quantitative analysis of vesicle clogs $>0.7 \mu \mathrm{m}$ along a length $50 \mu \mathrm{m}$ long. Statistical significance for all experiments was calculated using an unpaired Student's $t$ test: ${ }^{*} p<0.05 . n=15$.


Figure 3. Tip60 HAT activity loss in the CNS does not impact tubulin acetylation status in the motor axons where vesicle stalling takes place. Confocal imaging analysis of motor neurons immunohistochemically double-labeled with HRP (green) and anti-acetylated $\alpha$-tubulin antibody (red) that specifically labels acetylated MTs. $A$, Merged image of HRP and acetylated $\alpha$-tubulin antibody double-labeled control $w^{1118}$ larvae. $\boldsymbol{B}$, Acetylated $\alpha$-tubulin antibody labeled control $w^{1718}$ larvae. C, Merged image of HRP and acetylated $\alpha$-tubulin antibody double-labeled dTip $60{ }^{\mathrm{E} 4310} \mathrm{~B}$ larvae. $\boldsymbol{D}$, Acetylated $\alpha$-tubulin antibody labeled dTip $60{ }^{\mathrm{E} 4310} \mathrm{~B}$ larvae. $E$, Histogram depicting quantitation of the mean fluorescence intensity of anti-acetylated tubulin staining in dTip6 ${ }^{\mathrm{E} 4310}$ and control $w^{1718}$ fly lines. Statistical significance for all experiments was calculated using an unpaired Student's $t$ test: $p<0.05 . N=15$.

uitous induction of Tip60 ${ }^{\mathrm{E} 431 \mathrm{Q}}$ in whole larvae (Lorbeck et al., 2011) to specifically identify axonal transport-linked genes that are misregulated in response to Tip60 HAT loss. Our analysis using the Panther classification system identified 34 genes that were functionally linked to axonal transport (Table 1). Among these, genes that encode members of Kinesin and Dynein superfamilies of motor proteins were highly represented. Intriguingly, many of the identified genes were associated with $\mathrm{AD}$, Parkin- 
Table 1. Axonal transport pathways significantly misregulated because of dTip60 HAT loss

\begin{tabular}{lc}
\hline Axonal transport-related pathway & Number of genes \\
\hline Kinesin family & 5 \\
Cytoplasmic dynein or dynactin & 5 \\
Accessory genes required for axonal transport & 7 \\
Microtubule-associated protein & 6 \\
Non-motor actin-binding protein & 2 \\
Membrane-trafficking regulatory protein & 10 \\
Alzheimer's disease-presenilin pathway & 6 \\
Parkinson's disease & 4 \\
Huntington's disease & 8 \\
Notch signaling pathway & 3 \\
Integrin signaling pathway & 6 \\
Synaptic transmission & 15 \\
Axon guidance & 8 \\
Cytoskeletal organization/regulation & 13 \\
\hline
\end{tabular}

son's disease, and Huntington's disease, all neurodegenerative disorders in which aggregation of vesicles resulting from axonal transport stalling and locomotor dysfunction is a common characteristic (Roy et al., 2005; Duncan and Goldstein, 2006).

To extend and confirm our microarray analysis, we performed quantitative real-time PCR analysis of six of these genes (Fig. 4A) that were representative of a particular pathway or protein family member (Fig. 4B), this time using staged dTip $60^{\mathrm{E} 431 \mathrm{Q}}$ third instar brain tissue under the control of the elav-GAL4 driver. Khc (Kinesin heavy chain) and Klp64D (Kinesin-like protein at 64D) were two confirmed gene targets significantly upregulated and downregulated, respectively, in response to Tip60 HAT loss that have roles in anterograde transport (Hurd and Saxton, 1996; Ray et al., 1999). Additional upregulated targets included genes with established roles in retrograde transport, such as Dhc64C (Dynein heavy chain 64C) and Glued $\left(p 150^{\text {glued }}\right)$, a dynactin component that serves as an adaptor to link cargo to dynein for transport. Similar to our Tip60 mutant phenotypes, abnormal activation of cytoplasmic dynein heavy chain (Dhc64C) and the $p 150^{\text {Glued }}$ (Glued) component of the dynactin complex results in axonal stalling and posterior paralysis (Martin et al., 1999). Downregulated targets with confirmed roles in axonal vesicle transport included Synaptotagmin 1 (Syt1), an integral synaptic vesicle protein required for vesicular trafficking, exocytosis, and neurotransmitter release (Martin et al., 1999), and $A p p l$, the Drosophila homolog of human App. Intriguingly, both reduction and excess Appl and App in flies cause axonal vesicle transport stalling and locomotor defects almost identical to the phenotypes we observed in our Tip60 HAT mutant larvae (Torroja et al., 1999; Gunawardena and Goldstein, 2001). In summary, our identification of axonal vesicle transport and locomotor linked genes that are misregulated in the larval brain in response to Tip60 HAT loss support a transcriptional regulatory role for dTip60 in these neuronal processes.

\section{Certain HDACi rescue both locomotor dysfunction and axonal vesicle accumulations in Tip60 HAT mutant larvae} The effects of Tip60 HAT reduction on locomotion and axonal transport prompted us to investigate what effect an HDAC inhibitor might have on the flies. We hypothesized that, if reduction of Tip60-induced acetylation levels in dTip60 ${ }^{\mathrm{E} 431 \mathrm{Q}}$ HAT mutant larvae is a mechanism underlying axonal transport defects, then an HDACi could partially rescue these defects by maintaining and/or increasing global acetylation levels in these Tip60 HAT mutant flies. To test this hypothesis, larvae were reared on food containing one of seven types of HDACi (ms-275, sodium butyrate, SAHA, Givinostat, valproic acid, Romidepsin, or Mocetinostat). To select an appropriate concentration of drug that was not toxic to the larvae, dose-response curve experiments were performed on wild-type larvae using different titrations of each drug and the highest concentration of drug that caused no observable locomotion defects in wild-type larvae was selected for use.

Interestingly, although all seven types of HDACi tested were classified as inhibitors of either class I or both class I and II HDACs, only treatment of larvae with ms-275, Givinostat, or Mocetinostat showed significant rescue for locomotor ability as assessed by line crossing and contraction ability assays (Fig. $5 A, B)$. Moreover, only larvae treated with ms-275, Givinostat, and Mocetinostat showed rescue of the axonal transport phenotype (Fig. $5 C-G$ ). These results reveal that, although the inhibitors tested were classified as similar in terms of the HDAC classes they target (Chuang et al., 2009), they showed differences in their efficacy for treatment of axonal vesicle stalling and corresponding behavioral defects in vivo.

\section{Ms-275 treatment restores histone $\mathrm{H} 4$ chromatin acetylation levels at Tip60 misregulated target genes in Tip60 HAT mutant larvae}

The rescue of the locomotor function and axonal vesicle accumulations by selective HDAC treatment prompted us to ask whether a possible mechanism for the drug's action was via rescue of axonal transport-linked genes that were misexpressed in response to Tip60 HAT loss. To address this question, we performed quantitative real-time PCR analysis on the six confirmed Tip60 targets we had identified (Fig. 4B), this time using staged dTip $60^{\mathrm{E} 431 \mathrm{Q}}$ third instar brain tissue dissected from larvae reared on ms-275. For these and all subsequent studies, we opted to use ms-275 treatment as it showed strong efficacy in axonal defect treatment and is the most well-characterized drug we tested in terms of its nervous system specificity (Simonini et al., 2006). Of the six genes tested, mRNA levels of Appl and Syt1 in ms-275-treated Tip60 ${ }^{\mathrm{E} 431 \mathrm{Q}}$ expressing brains showed no significant difference compared with wild-type levels (Fig. 6A), indicating that ms-275 rescued expression of each of these genes. Together, these results demonstrate that treatment of larvae expressing dTip $60^{\mathrm{E} 431 \mathrm{Q}}$ in the nervous system with the brainspecific HDACi ms-275 rescues both locomotion phenotypes and axonal vesicle accumulations, possibly by inducing the expression levels of at least some genes important in regulating these processes.

The six genes were further analyzed to establish possible mechanisms underlying their transcriptional regulation. As each of these genes was misexpressed because of Tip60 HAT loss, we first asked whether these candidate genes were indeed direct targets of Tip60 in wild-type control flies $\left(w^{1118}\right)$ and whether Tip60 recruitment to these gene loci was affected in dominant-negative Tip60 HAT mutant flies (dTip60 ${ }^{\mathrm{E} 431 \mathrm{Q}}$ ). To address these questions, chromatin was isolated from larval anterior head regions from each of these genotypes and ChIP-qPCR analysis was used to detect in vivo binding of Tip60 at each of these gene loci. This analysis revealed significant enrichment for Tip60 binding in vivo at four of the six gene loci tested in control $w^{1118}$ larvae, thus classifying these genes ( $A p p l, D h c 64 C, K h c$, and Syt1) as novel direct Tip60 targets and Glued and $K l p 64 D$ as indirect Tip60 targets (Fig. 6B). In contrast, dTip60 ${ }^{\mathrm{E} 431 \mathrm{Q}}$ larvae showed no enrichment for Tip60 binding at any of these gene loci (Fig. 6B). 


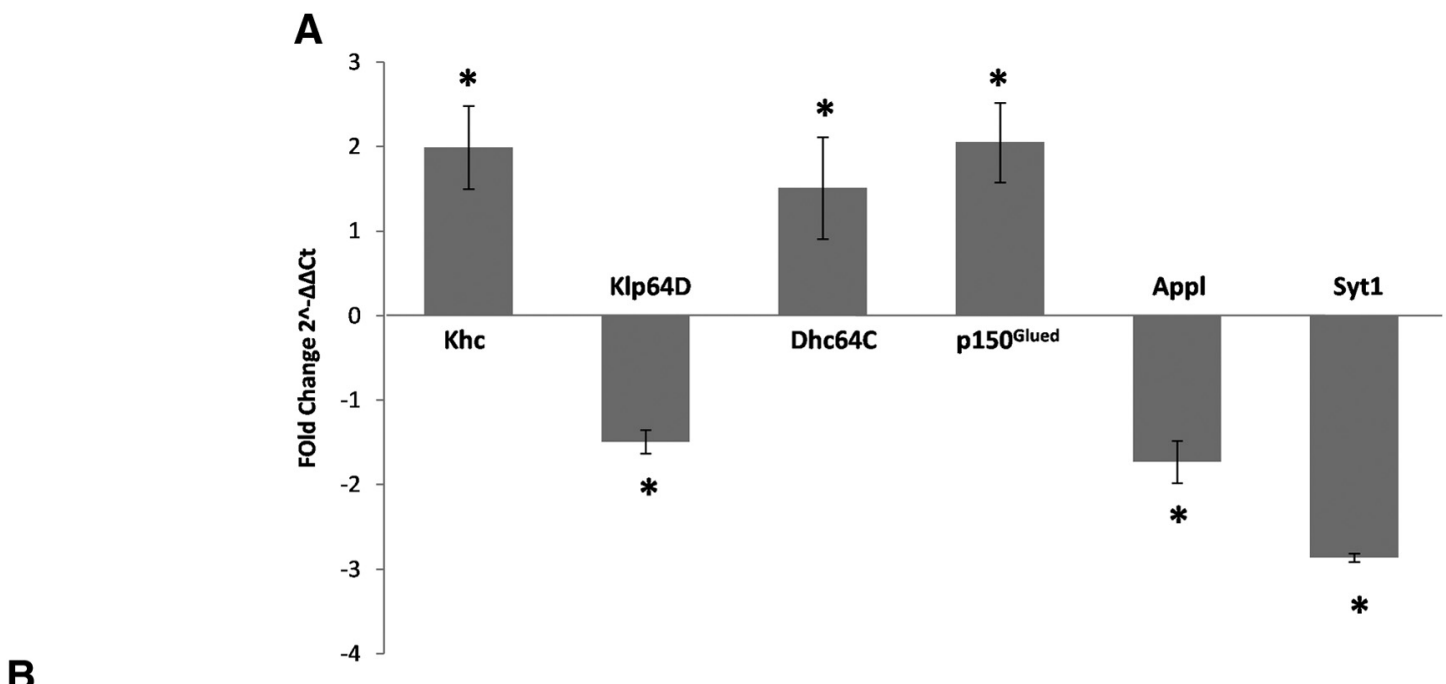

B

\begin{tabular}{|c|c|c|c|c|}
\hline $\begin{array}{l}\text { Gene } \\
\text { Name }\end{array}$ & Common Name & $\begin{array}{l}\text { PCR Fold } \\
\text { Change }\end{array}$ & Phenotype/Disease & Inferred Function \\
\hline Khc & Kinesin heavy chain & 1.99 & $\begin{array}{l}\text { axonal swellings containing vesicles, mitochondria, and } \\
\text { organelles }\end{array}$ & $\begin{array}{c}\text { anterograde axonal } \\
\text { transport }\end{array}$ \\
\hline KIp64D & Kinesin-like protein at 64D & -1.49 & axonal accumulation of AchE and ChAT & $\begin{array}{c}\text { axonal transport of AchE and } \\
\text { ChAT }\end{array}$ \\
\hline Dhc64C & Dynein heavy chain & 1.51 & mislocalization of Syt, CSP & retrograde axonal transport \\
\hline p150 Glued & p150glued & 2.05 & larval paralysis, accumulation of Syt in axons & retrograde axonal transport \\
\hline Appl & $\begin{array}{l}\text { Amyloid protein precursor- } \\
\text { like }\end{array}$ & -1.73 & synaptic vesicle accumulations & linker protein for Kinesin \\
\hline Syt1 & Synaptotagmin 1 & -2.86 & synaptic vesicle accumulations & calcium sensor \\
\hline
\end{tabular}

Figure 4. Loss of Tip60 HAT activity in the CNS causes misregulation of axonal transport genes. A, Real-time PCR was performed on cDNA isolated from staged third instar larval dissected brain tissue from larvae expressing dTip60 ${ }^{\mathrm{E} 4310}$ under the elav ${ }^{\mathrm{C} 155}$ pan-neuronal GAL4 driver. Histogram represents relative fold change in expression level of axonal transport-related target genes in brain of larvae expressing dTip60 ${ }^{\mathrm{E} 4310} \mathrm{~B}$. Real-time PCRs were performed in triplicate, and the fold change was calculated using the $\Delta \Delta C \mathrm{CT}$ method using RP49 as control. Statistical significance was calculated using an unpaired Student's $t$ test: ${ }^{*} p<0.05$. $\boldsymbol{B}$, List of selected axonal transport-related target genes and their functions that are identified in microarray analysis and validated in larval brain tissue using qRT-PCR.

Regulation of histone acetylation levels is a key mechanism by which Tip60 and HDACi exert their action on gene control. Therefore, we hypothesized that acetylation levels at Tip60 direct target genes would be enhanced in control $w^{1118}$ flies compared with dTip $60^{\mathrm{E} 431 \mathrm{Q}}$ where Tip60 recruitment is lacking, and that treatment of dTip $60^{\mathrm{E} 431 \mathrm{Q}}$ flies with ms-275 HDACi would restore acetylation levels at these targets genes. To test this, we assessed enrichment levels of pan-acetylated histone $\mathrm{H} 4$, which is the preferential histone target of Tip60, at each gene loci in $w^{1118}$, dTip $60^{\mathrm{E} 431 \mathrm{Q}}$, and ms-275-treated dTip $60^{\mathrm{E} 431 \mathrm{Q}}$ larvae in chromatin isolated from larvae heads. We found that $\mathrm{H} 4$ acetylation enrichment levels at all Tip60 target genes were enhanced in the $w^{1118}$ flies and significantly decreased in dTip $60^{\mathrm{E} 431 \mathrm{Q}}$ flies (Fig. $6 C$ ). As predicted, treatment of dTip $60^{\mathrm{E} 3431 \mathrm{Q}}$ larvae with ms-275 partially rescues acetylation loss at all but one $(A p p l)$ of the genes (Fig. 6C). Of note, we did observe enrichment of $\mathrm{H} 4$ acetylation at Tip60 indirect targets (Glued, Klp64D) relative to that found in dTip $60^{\mathrm{E} 431 \mathrm{Q}}$ larvae, suggesting that perhaps Tip60 may indirectly lead to increased $\mathrm{H} 4$ acetylation or nonenrichment for Tip60 site-specific acetylation at these sites is masked by the global pan-acetylation $\mathrm{H} 4$ antibodies used in these studies. Together, these results demonstrate that reduction of Tip60 HAT activity led to reduced histone H4 acetylation levels at Tip60 gene targets that can be partially rescued by treatment with the ms-275 HDACi.

Tip60 and APP functionally interact in the nervous system to mediate locomotion ability and axonal vesicle transport

We previously reported that Tip60 and APP functionally interact to mediate nervous system development and apoptotic cell death in the CNS of a Drosophila AD model (Pirooznia et al., 2012b). Given our finding that APPL expression is downregulated in the larval brain in response to Tip60 HAT loss and that Tip60 and APP misexpression each separately causes similar defects in axonal vesicle transport in larvae, we hypothesized that these proteins might act together to mediate this process. To test this hypothesis, we used unique UAS-responsive transgenic fly lines generated in our laboratory (Pirooznia et al., 2012b) that coexpress equivalent levels of dTip $60^{\mathrm{E} 431 \mathrm{Q}}$ and APP or dTip60 ${ }^{\mathrm{E} 431 \mathrm{Q}}$ and APP dCT (APP lacking the $\mathrm{C}$ terminus Tip60 interacting domain). Functional behavior analysis of these larvae using locomotion assays revealed a synergistic interaction between Tip60 and APP as coexpression of dTip $60^{\mathrm{E} 431 \mathrm{Q}}$ with APP resulted in locomotion phenotypes nearly fourfold relative to that observed in larvae expressing APP or dTip60 ${ }^{\mathrm{E} 431 \mathrm{Q}}$ alone (Fig. $7 A-C$ ). These fly lines were then crossed to flies containing the 


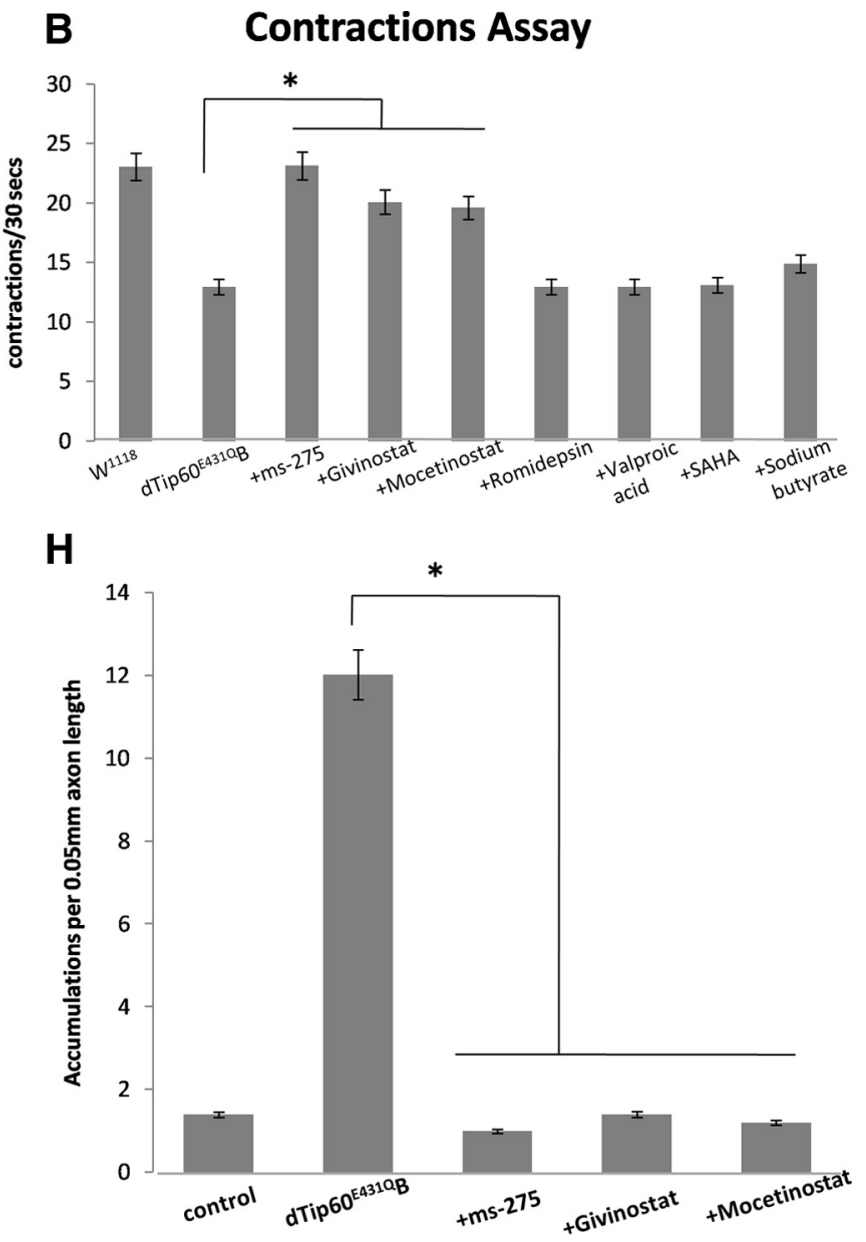

Figure 5. Certain HDACi rescue both locomotor dysfunction and axonal vesicle accumulations in Tip60 HAT mutant larvae. Third instar larvae expressing dTip60 ${ }^{\text {E4310 }} \mathrm{B}$ under the control of the elav ${ }^{\mathrm{C} 155}$ pan-neuronal GAL4 driver were raised on food containing no drug or each of the following HDACi: ms-275, sodium butyrate, SAHA, Givinostat, valproic acid, Romidepsin, or Mocetinostat. A, Histogram represents results of the line crossing assay. Only the following HDACs showed significant rescue, with ms-275 treatment showing $77 \%$ rescue, Givinostat treatment showing $96 \%$ rescue, and Mocetinostat treatment showing $95 \%$ rescue. $\boldsymbol{B}$, Contraction assay. Only the following HDACs showed significant rescue, with ms-275 treatment showing $100 \%$ rescue, Givinostat treatment showing $87 \%$ rescue, and Mocetinostat treatment showing $85 \%$ rescue. $N=30 . p<0.05$. C $-G$, Representative confocal images of motor axons directly posterior to brains in third instar larvae coexpressing a synaptotagmin-e-GFP fusion protein (syt-eGFP) along with dTip60 ${ }^{\mathrm{E} 4310}$ under the control of the pan-neuronal elav-GAL4 driver reared on food treated with either no drug, ms-275, Givinostat, or Mocetinostat. $\boldsymbol{H}$, Histogram represents quantitative analysis of vesicle clogs $>0.7 \mu \mathrm{m}$ along a length $50 \mu \mathrm{m}$ long. Statistical significance was calculated using an unpaired Student's $t$ test: ${ }^{*} p<0.05 . N=10$.

synaptotagmin-e-GFP fusion protein transgene (syt-eGFP) under the control of the pan-neuronal elav-GAL4 driver to track axonal vesicle transport in response to misexpression of both Tip60 and APP (Zhang et al., 2002). Confocal imaging analysis of motor axons was used to assess the distribution of GFP-tagged axonal vesicles in the motor axons extending from the CNS in third instar larvae. Consistent with the above result, quantitative analysis of vesicle accumulations in larvae overexpressing both dTip $60^{\mathrm{E} 431 \mathrm{Q}}$ and APP revealed an exacerbated phenotype indicative of a synergistic genetic interaction between the two proteins (Fig. 8I). Vesicle accumulations were approximately fourfold higher in larvae coexpressing dTip $60{ }^{\mathrm{E} 431 \mathrm{Q}}$ and APP than in larvae expressing dTip $60^{\mathrm{E} 431 \mathrm{Q}}$ or APP alone (Fig. $8 B, C, E$ ). Importantly, and as we predicted, the Tip60 and APP interaction was dependent upon the $\mathrm{C}$ terminus of APP that interacts with Tip60 (Cao and Sudhof, 2001) as coexpression of dTip $60^{\mathrm{E} 431 \mathrm{Q}}$ and APP dCT resulted in a moderate level of vesicle accumulations and locomotor dysfunction that was approximately equivalent to that observed for dTip $60^{\mathrm{E} 431 \mathrm{Q}}$ alone (Figs. $7 A-C$ and $8 B, D, F$ ).

We previously demonstrated that dTip60 displays a neuroprotective role in $\mathrm{AD}$ neurodegenerative pathology in that in- creased levels of dTip60 HAT activity can suppress APP-induced apoptotic cell death in the CNS of a fly AD model (Pirooznia et al., 2012b). These findings prompted us to ask whether elevated Tip60 HAT levels would also suppress the APP-induced axonal transport and locomotor-defective phenotypes. To address this question, we performed axonal transport assays and functional larval locomotion assays in third instar larvae coexpressing both dTip60 ${ }^{\mathrm{WT}}$ and APP using our APP;dTip60WT line C that expresses the highest levels of wild-type Tip60 for all of our dTip60 ${ }^{\text {WT }}$ lines (Pirooznia et al., 2012b). Remarkably, we found that additional levels of Tip60 partially rescued APP-induced vesicle aggregation as evidenced by a significant reduction of the presence of vesicle accumulations in the motor neurons of larvae expressing both APP and dTip60 ${ }^{\mathrm{WT}}$ compared with APP alone (Fig. $8 B, G, H$ ). Functional analysis of the Tip60 and APP interaction using locomotion assays demonstrated that excess levels of dTip $60^{\mathrm{WT}}$ also partially rescued APP-induced locomotor defects (Fig. 7A-C). Importantly, and as we predicted, partial rescue of APP-induced axonal vesicle transport and locomotor defects was dependent upon the Tip60 interacting $C$ terminus of APP, as larvae coexpressing both dTip60 ${ }^{\mathrm{WT}}$ and APP-dCT do not show 

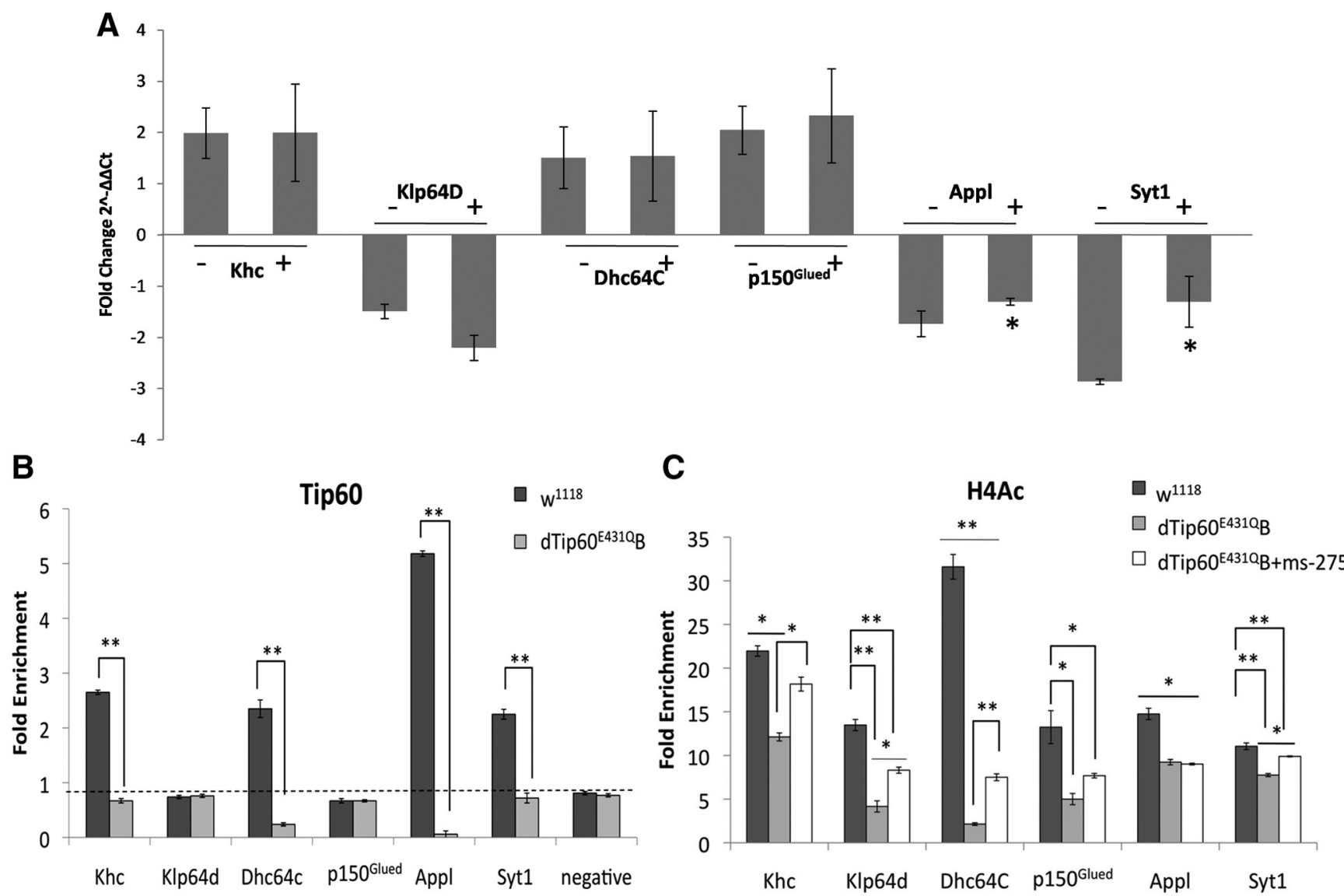

Figure 6. ChIP enrichment of target genes by Tip60 and pan-acetylated histone $H 4$ antibodies. A, Real-time PCR was performed on cDNA isolated from staged third instar larval dissected brain tissue from either ms-275-treated or untreated larvae expressing dTip60 ${ }^{\mathrm{E} 4310}$ using the elav ${ }^{\mathrm{C} 155}$ pan-neuronal GAL4 driver. Each histogram bar represents the fold change in expression level of axonal transport-related target genes in brains of larvae expressing either dTip60 ${ }^{\mathrm{E} 4310} \mathrm{~B}$ or $\mathrm{dTip} 60^{\mathrm{E} 4310} \mathrm{~B}$ larvae treated with ms-275 relative to $\mathrm{w}^{1118}$ control flies. Real-time PCRs were performed in triplicate, and the fold change was calculated using the $\Delta \Delta C_{\mathrm{t}}$ method using RP49 as control. Asterisks above Appl and Syt 1 denote significant fold changes in gene targets between treated larvae and untreated larvae, indicating partial rescue of expression with ms-275 for these genes. Statistical significance was calculated using an unpaired Student's $t$ test: ${ }^{*} p<0.05 . \boldsymbol{B}_{\text {, Chromatin was }}$ isolated from 300 pooled larvae heads for each of the indicated genotypes. (hIP was performed using ChIP-IT Express Kit (Active Motif) using Tip60 antibodies and (C) pan-acetylated histone H4 antibodies. For each ChIP experiment, a mock reaction containing no antibody was performed simultaneously as a negative control. Real-time PCR was performed on DNA purified from each of the ChIP reactions using primer pairs specific for each gene loci. Fold enrichment of the respective genes was calculated relative to the mock no antibody control using the standard curve method as described in the ChIP-IT Express Kit manual. $A$, Dotted line represents baseline enrichment level set by using negative control primers that amplify a fragment within a gene desert within Drosophila chromosome 3L. All data are from three independent qPCR experiments for each genotype. Statistical significance for all experiments was calculated using an unpaired Student's $t$ test: ${ }^{*} p \leq 0.05$; ${ }^{* *} p \leq 0.01$. Values are mean \pm SEM.

this rescue (Fig. $7 A-C$ ). Together, these results demonstrate that Tip60 and APP functionally interact to mediate motor axon vesicle transport and locomotor function in larvae and that this interaction is dependent upon the Tip60 interacting $\mathrm{C}$ terminus of APP.

\section{Discussion}

Previously, we demonstrated that Tip60 HAT activity mediates APP-induced lethality and apoptotic cell death in the CNS of an AD fly model (Pirooznia et al., 2012b). Here, we further examine this functional interaction in neuronal development by testing the hypothesis that Tip60 and APP functionally interact to mediate axonal synaptic vesicle transport, a process shown to involve APP itself (Torroja et al., 1999; Gunawardena and Goldstein, 2001; Stokin et al., 2005). We show that loss of dTip60 HAT function in the CNS causes synaptic vesicle accumulations in motor axons extending from the CNS and associated locomotor function defects, supporting a role for Tip60 HAT activity in these processes. Further, we found that Tip60 HAT mutantinduced locomotion and transport defects are exacerbated by APP, whereas excess Tip60 rescues APP-mediated axonal trans- port defects, supporting a functional interaction between these proteins in these processes. Directional motor-dependent trafficking is thought to be controlled, at least in part, by acetylation of $\alpha$-tubulin within MTs that is associated with stable MT and serves as a guidance cue to allow for polarity determination (Janke and Kneussel, 2010). In this regard, we previously reported that Tip60 HAT loss within the fly neuromuscular junction results in reduction of acetylated MTs only at ends of axons specifically extending into the terminal synaptic boutons in conjunction with defects in bouton growth and development (Sarthi and Elefant, 2011). However, we detected no changes in the $\alpha$-tubulin acetylation levels along the entire length of the motor axons in dTip $60^{\mathrm{E} 431 \mathrm{Q}}$ larvae where vesicle clogging is observed, suggesting that Tip60 mediates its function in vesicle transport, at least in part, via epigenetic control of axonal transport-linked genes. In support of this concept, we identified a number of axonal transport-linked genes that were misregulated in response to dTip60 HAT loss. Inappropriate Tip60/APP-AICD complex formation and/or its recruitment to chromatin may contribute to $\mathrm{AD}$-linked neurodegeneration by causing aberrant epigenetic 



Figure 7. Tip60 and APP functionally interact in the nervous system to mediate locomotion ability. Histograms representing the results of a series of locomotion assays conducted on $w^{1118}$ control third instar larvae or third instar larvae expressing each of the different GAL4 responsive transgenes under the control of the elav ${ }^{C 155}$ pan-neuronal GAL4 driver for the following assays. $A$, Line crossing assay. $\boldsymbol{B}$, Contraction assay. C, Righting assay. Statistical significance for all experiments was calculated using an unpaired Student's test: ${ }^{*} p<0.05 . n=30$.

marks with subsequent negative effects on neuronal gene expression profiles (Baek et al., 2002; Hebert et al., 2006). In support of this concept, we recently reported that expression of certain $\mathrm{AD}$ linked Tip60 gene targets critical for regulating apoptotic pathways is modified by excess APP in an AD fly model (Pirooznia et al., 2012b). Thus, our results support a model for APP in inducing axonal transport defects via misregulation of the Tip60/AICD complex required for transcriptional regulation of Tip60 genes linked to transport function, such as motor proteins.

Loss of HAT activity in the CNS is accompanied by transcriptional dysfunction that causes neuronal malfunction (Levenson and Sweatt, 2005) and is associated with a number of disorders of human cognition, including Rubinstein-Taybi syndrome (Petrij et al., 1995), schizophrenia (Chen et al., 2002), and more recently, AD (Pirooznia et al., 2012b). Numerous studies have examined the efficacy of HDACi as therapeutics for treatment of cognitive disorders. HDACi often upregulate general acetylation to relieve transcriptional repression (Kim et al., 2000; Richon et al., 2000), which facilitates cell survival. Specific HDACi have been shown to partially rescue dendritic spine reduction associated with memory loss in a mouse model of $\mathrm{AD}$ as well as expression of several plasticity-related proteins (Ricobaraza et al., 2012). However, although advances have been made to design more selective $\mathrm{HDACi}$, there is some speculation about the target specificity of $\mathrm{HDACi}$ as they predominantly function by inhibiting classes of proteins, some with multiple nonredundant functions (Selvi et al., 2010). Thus, the current use of pan-HDACi that act by increasing global acetylation levels can also disrupt cellular acety- lation homeostasis with subsequent negative consequences. Moreover, targeting a particular class of HDACs is an arduous task as the causative agents of memory impairing histone acetylation changes and hence, the best targets for pharmacological strategies, remain unknown (Graff et al., 2012). Here, we test the efficacy of different HDACi in treating axonal defects, in vivo, by screening a panel of seven different HDACi (Fig. 5). Interestingly, although all of these inhibitors are classified to predominantly target either class I or classes I and II HDACs, only three of the HDACs we tested were able to rescue the locomotion and axonal defects we observed in Tip60 HAT mutant larvae, supporting their specificity in terms of efficacy of axonal defect treatment as well as the value of testing these drugs on specific neuronal defects using an in vivo model system.

The steady-state level of histone acetylation in the brain is dynamically maintained by the opposing actions of HATs and HDACs that are critical for transcriptional regulation and that must be stringently maintained. Alterations that disrupt the HAT/HDAC balance, and thus acetylation levels, cause transcriptional dysregulation that alters cellular homeostasis (Langley et al., 2005). Consistent with these studies, our findings suggest that axonal defects are influenced by changes in expression of axonal transport-linked genes in Tip60 HAT mutant larvae (Fig. 4). Indeed, we confirmed that four (Appl, Dhc64c, Khc, Syt1) of the six axonal transport genes were novel bona fide Tip60 direct targets as Tip60 in vivo binding was detected at their loci. In contrast, Tip60 binding was not detected at any of the gene loci in the Tip60 HAT mutant larvae with concomitant reduction of chro- 

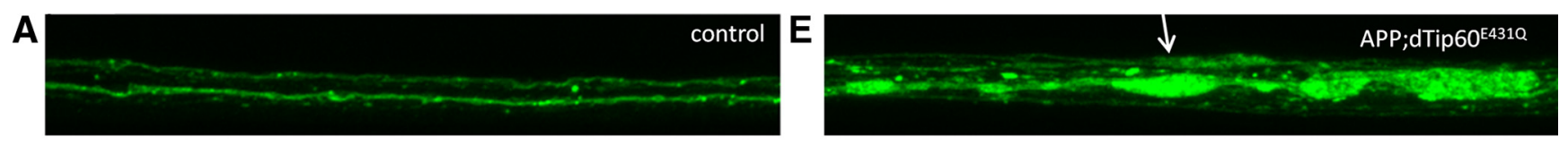

B
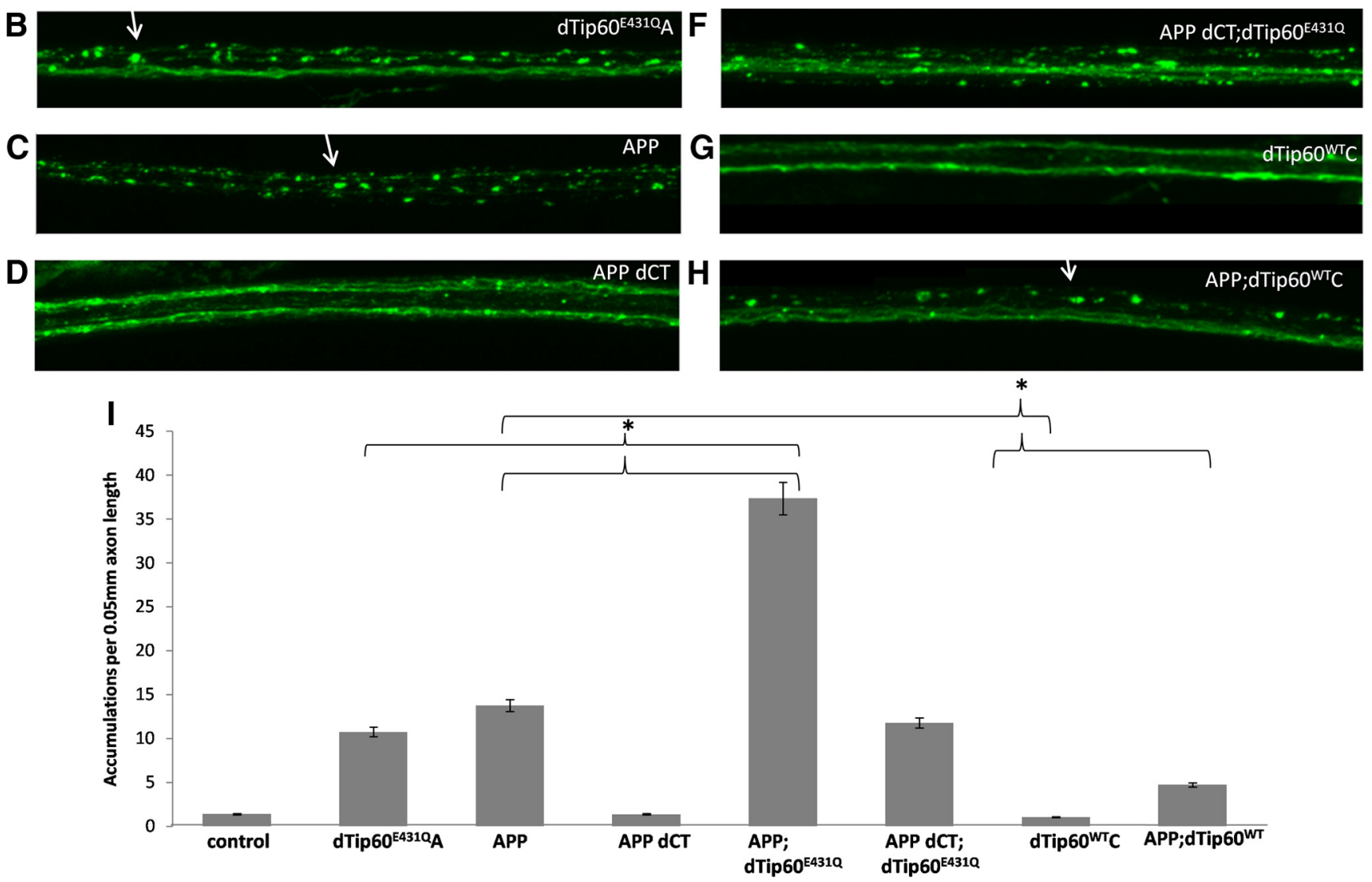

Figure 8. Tip60 and APP functionally interact in the nervous system to mediate axonal vesicle transport. Representative confocal images of larval motor axons directly posterior to the brain of third instar larvae expressing indicated transgenes driven in the nervous system by the elav ${ }^{\mathrm{C} 155}$ pan-neuronal GAL4 driver expressing synaptotagmin fused with eGFP. A, Control $w^{1118}$ larvae show no aggregates, whereas $(\boldsymbol{B})$ larvae expressing dTip60 ${ }^{\mathrm{E} 4310}$ show significantly more aggregates than wild-type control. $\boldsymbol{C}$, Full-length APP causes axonal accumulations, whereas (D) no aggregates were observed in larvae expressing a truncated version of APP lacking the C-terminal domain. $\boldsymbol{E}$, Coexpression of Tip60 ${ }^{\mathrm{E} 3310}$ and APP results in exacerbated axonal accumulations that is $(\boldsymbol{F})$ dependent on expression of the $\left(\right.$ terminus of APP. G, Larvae expressing excess Tip60 ${ }^{\text {WT }}$ show no accumulations, whereas $(\boldsymbol{H})$ coexpression of dTip60 ${ }^{\text {WT }}$ with APP partially rescues axonal stalling phenotype. Line arrows indicate synaptotagmin-positive vesicle aggregations. I, Histogram represents quantitative analysis of vesicle clogs $>0.7 \mu \mathrm{m}$ along a length $50 \mu \mathrm{m}$ long. Statistical significance for all experiments was calculated using an unpaired Student's $t$ test: ${ }^{*} p<0.05 . n=15$.

matin acetylation at histone $\mathrm{H} 4$ at these same sites. These results indicate that the HAT mutation in Tip60 both reduces endogenous histone H4 acetylation levels (Lorbeck et al., 2011) and negatively impacts recruitment of both HAT mutant and endogenous wild-type Tip60 to its target genes. Interestingly, although we found that treatment with $\mathrm{ms}-275$ enhances histone $\mathrm{H} 4$ acetylation levels partially back to control levels at the majority of the genes tested, only two of these genes showed partial transcriptional rescue (Fig. 6A). Conversely, although Khc was the only gene found to show full acetylation rescue at histone $\mathrm{H} 4$ by ms275 treatment, it was not one of the genes that was transcriptionally rescued. Such partial rescue is consistent with our finding that, although select HDACi fully rescued axonal stalling, they only partially rescued the more complex associated behavioral locomotion phenotypes. Such partial rescue of disease-linked morphology and target gene expression by an HDACi is not without precedent, as previous studies have concluded that, although HDACi treatment induces general hyperacetylation, it is often not sufficient to produce absolute beneficial effects alone. For instance, Vecsey et al. (2007) demonstrated that general induction of histone acetylation by HDACi treatment was not suffi- cient to rescue synaptic plasticity and memory loss in the hippocampus of a mouse model lacking the learning and memory-linked HAT CBP, and that a wild-type CBP allele in conjunction with HDACi treatment was required. Such incomplete rescue by HDACi treatment alone is likely because induction of certain synaptic plasticity target genes still requires recruitment of CBP. Consistent with these findings, our results suggest that global enhancement of histone $\mathrm{H} 4$ acetylation by select HDACi treatment is not sufficient for full rescue of certain genes, as they may still require recruitment of Tip60 for sitespecific acetylation and/or for interaction with additional transcriptional factors.

Many HATs, as opposed to HDACs, have nonredundant physiological functions and are required for site-specific chromatin marks that promote specific gene expression profiles (Rouaux et al., 2003). These findings support the notion of exploring the efficacy of specific HAT modulators for use in therapeutic treatment in conjunction with HDACi, as they would have more direct and specific effects over more general HDACi treatment (Selvi et al., 2010). Indeed, the HAT CBP has been shown to play a neuroprotective role in neurodegenerative processes linked to 
multiple cognition linked disorders (Rouaux et al., 2004). Moreover, partial loss of Tip60 has recently been reported to slow the progression of spinocerebellar ataxia (Gehrking et al., 2011). Here, we report that, although Tip60 overexpression alone causes no observable axonal transport phenotypes, its excess production in conjunction with APP partially rescues both APP-mediated behavioral and axonal transport defects as well as APP-mediated apoptotic neuronal cell death in the CNS and axonal growth that regulates sleep (Pirooznia et al., 2012a, 2012b). Thus, our data support an all-encompassing neuroprotective function for Tip60 in multiple neuronal processes that are interrupted in AD pathology. We propose that Tip60 might exert this neuroprotective function either by itself or by complexing with other peptides, such as AICD, for its recruitment and site-specific acetylation of specific neuronal gene promoters to redirect their expression and function in selective neuronal processes, such as axonal transport. Consistent with this concept, our previous study supports a model by which Tip60 and or Tip60/AICD act to induce neuroprotective and developmental genes that act together to skew cell fate from apoptotic cell death toward cell survival under neurodegenerative conditions, such as excess APP (Pirooznia et al., 2012b). Further elucidation of the fundamental role of Tip60 in neuronal processes linked to neurodegenerative diseases, such as $\mathrm{AD}$, should help guide the generation and usage of specific HAT modulators for the treatment of cognitive disorders.

\section{References}

Baek SH, Ohgi KA, Rose DW, Koo EH, Glass CK, Rosenfeld MG (2002) Exchange of N-CoR corepressor and Tip60 coactivator complexes links gene expression by NF- $\kappa \mathrm{B}$ and $\beta$-amyloid precursor protein. Cell 110 : 55-67. CrossRef Medline

Budnik V, Ruiz-Canada C (2006) The fly neuromuscular junction: structure and function, Ed 2. New York: Elsevier.

Cao X, Südhof TC (2001) A transcriptionally active complex of APP with Fe65 and histone acetyltransferase Tip60. Science 293:115-120. CrossRef Medline

Carrozza MJ, Utley RT, Workman JL, Côté J (2003) The diverse functions of histone acetyltransferase complexes. Trends Genet 19:321-329. CrossRef Medline

Chen Y, Sharma RP, Costa RH, Costa E, Grayson DR (2002) On the epigenetic regulation of the human reelin promoter. Nucleic Acids Res 30: 2930-2939. CrossRef Medline

Chuang DM, Leng Y, Marinova Z, Kim HJ, Chiu CT (2009) Multiple roles of HDAC inhibition in neurodegenerative conditions. Trends Neurosci 32:591-601. CrossRef Medline

Duncan JE, Goldstein LS (2006) The genetics of axonal transport and axonal transport disorders. PLoS Genet 2:e124. CrossRef Medline

Gehrking KM, Andresen JM, Duvick L, Lough J, Zoghbi HY, Orr HT (2011) Partial loss of Tip60 slows mid-stage neurodegeneration in a spinocerebellar ataxia type 1 (SCA1) mouse model. Hum Mol Genet 20:2204-2212. CrossRef Medline

Gindhart JG Jr, Desai CJ, Beushausen S, Zinn K, Goldstein LS (1998) Kinesin light chains are essential for axonal transport in Drosophila. J Cell Biol 141:443-454. CrossRef Medline

Gräff J, Rei D, Guan JS, Wang WY, Seo J, Hennig KM, Nieland TJ, Fass DM, Kao PF, Kahn M, Su SC, Samiei A, Joseph N, Haggarty SJ, Delalle I, Tsai LH (2012) An epigenetic blockade of cognitive functions in the neurodegenerating brain. Nature 483:222-226. CrossRef Medline

Gunawardena S, Goldstein LS (2001) Disruption of axonal transport and neuronal viability by amyloid precursor protein mutations in Drosophila. Neuron 32:389-401. CrossRef Medline

Hammond JW, Cai D, Verhey KJ (2008) Tubulin modifications and their cellular functions. Curr Opin Cell Biol 20:71-76. CrossRef Medline

Hébert SS, Serneels L, Tolia A, Craessaerts K, Derks C, Filippov MA, Müller U, De Strooper B (2006) Regulated intramembrane proteolysis of amyloid precursor protein and regulation of expression of putative target genes. EMBO Rep 7:739-745. CrossRef Medline

Hurd DD, Saxton WM (1996) Kinesin mutations cause motor neuron dis- ease phenotypes by disrupting fast axonal transport in Drosophila. Genetics 144:1075-1085. Medline

Janke C, Kneussel M (2010) Tubulin post-translational modifications: encoding functions on the neuronal microtubule cytoskeleton. Trends Neurosci 33:362-372. CrossRef Medline

Kim YB, Ki SW, Yoshida M, Horinouchi S (2000) Mechanism of cell cycle arrest caused by histone deacetylase inhibitors in human carcinoma cells. J Antibiot (Tokyo) 53:1191-1200. CrossRef Medline

Langley B, Gensert JM, Beal MF, Ratan RR (2005) Remodeling chromatin and stress resistance in the central nervous system: histone deacetylase inhibitors as novel and broadly effective neuroprotective agents. Curr Drug Targets CNS Neurol Disord 4:41-50. CrossRef Medline

Levenson JM, Sweatt JD (2005) Epigenetic mechanisms in memory formation. Nat Rev Neurosci 6:108-118. CrossRef Medline

Levenson JM, O’Riordan KJ, Brown KD, Trinh MA, Molfese DL, Sweatt JD (2004) Regulation of histone acetylation during memory formation in the hippocampus. J Biol Chem 279:40545-40559. CrossRef Medline

Livak KJ, Schmittgen TD (2001) Analysis of relative gene expression data using real-time quantitative PCR and the 2(-delta delta $\mathrm{C}(\mathrm{T})$ ) method. Methods 25:402-408. CrossRef Medline

Lorbeck M, Pirooznia K, Sarthi J, Zhu X, Elefant F (2011) Microarray analysis uncovers a role for Tip60 in nervous system function and general metabolism. PLoS One 6:14. CrossRef Medline

Martin M, Iyadurai SJ, Gassman A, Gindhart JG Jr, Hays TS, Saxton WM (1999) Cytoplasmic dynein, the dynactin complex, and kinesin are interdependent and essential for fast axonal transport. Mol Biol Cell 10:37173728. Medline

Mudher A, Shepherd D, Newman TA, Mildren P, Jukes JP, Squire A, Mears A, Drummond JA, Berg S, MacKay D, Asuni AA, Bhat R, Lovestone S (2004) GSK- $3 \beta$ inhibition reverses axonal transport defects and behavioural phenotypes in Drosophila. Mol Psychiatry 9:522-530. CrossRef Medline

Peleg S, Sananbenesi F, Zovoilis A, Burkhardt S, Bahari-Javan S, Agis-Balboa RC, Cota P, Wittnam JL, Gogol-Doering A, Opitz L, Salinas-Riester G, Dettenhofer M, Kang H, Farinelli L, Chen W, Fischer A (2010) Altered histone acetylation is associated with age-dependent memory impairment in mice. Science 328:753-756. CrossRef Medline

Petrij F, Giles RH, Dauwerse HG, Saris JJ, Hennekam RC, Masuno M, Tommerup N, van Ommen GJ, Goodman RH, Peters DJ (1995) RubinsteinTaybi syndrome caused by mutations in the transcriptional co-activator CBP. Nature 376:348-351. CrossRef Medline

Pirooznia SK, Chiu K, Chan MT, Zimmerman JE, Elefant F (2012a) Epigenetic regulation of axonal growth of Drosophila pacemaker cells by histone acetyltransferase tip60 controls sleep. Genetics 192:1327-1345. CrossRef Medline

Pirooznia SK, Sarthi J, Johnson AA, Toth MS, Chiu K, Koduri S, Elefant F (2012b) Tip60 HAT activity mediates APP induced lethality and apoptotic cell death in the CNS of a Drosophila Alzheimer's disease Model. PLoS One 7:e41776. CrossRef Medline

Ray K, Perez SE, Yang Z, Xu J, Ritchings BW, Steller H, Goldstein LS (1999) Kinesin-II is required for axonal transport of choline acetyltransferase in Drosophila. J Cell Biol 147:507-518. CrossRef Medline

Richon VM, Sandhoff TW, Rifkind RA, Marks PA (2000) Histone deacetylase inhibitor selectively induces p21WAF1 expression and geneassociated histone acetylation. Proc Natl Acad Sci U S A 97:10014-10019. CrossRef Medline

Ricobaraza A, Cuadrado-Tejedor M, Marco S, Pérez-Otaño I, García-Osta A (2012) Phenylbutyrate rescues dendritic spine loss associated with memory deficits in a mouse model of Alzheimer disease. Hippocampus 22: 1040-1050. CrossRef Medline

Rouaux C, Jokic N, Mbebi C, Boutillier S, Loeffler JP, Boutillier AL (2003) Critical loss of CBP/p300 histone acetylase activity by caspase- 6 during neurodegeneration. EMBO J 22:6537-6549. CrossRef Medline

Rouaux C, Loeffler JP, Boutillier AL (2004) Targeting CREB-binding protein (CBP) loss of function as a therapeutic strategy in neurological disorders. Biochem Pharmacol 68:1157-1164. CrossRef Medline

Roy S, Zhang B, Lee VM, Trojanowski JQ (2005) Axonal transport defects: a common theme in neurodegenerative diseases. Acta Neuropathol 109:5-13. CrossRef Medline

Rusu P, Jansen A, Soba P, Kirsch J, Löwer A, Merdes G, Kuan YH, Jung A, Beyreuther K, Kjaerulff O, Kins S (2007) Axonal accumulation of synaptic markers in APP transgenic Drosophila depends on the NPTY motif and is 
paralleled by defects in synaptic plasticity. Eur J Neurosci 25:1079-1086. CrossRef Medline

Sapountzi V, Logan IR, Robson CN (2006) Cellular functions of TIP60. Int J Biochem Cell Biol 38:1496-1509. CrossRef Medline

Sarthi J, Elefant F (2011) dTip60 HAT activity controls synaptic bouton expansion at the Drosophila neuromuscular junction. PLoS One 6:e26202. CrossRef Medline

Selvi BR, Cassel JC, Kundu TK, Boutillier AL (2010) Tuning acetylation levels with HAT activators: therapeutic strategy in neurodegenerative diseases. Biochim Biophys Acta 1799:840-853. CrossRef Medline

Simonini MV, Camargo LM, Dong E, Maloku E, Veldic M, Costa E, Guidotti A (2006) The benzamide MS-275 is a potent, long-lasting brain regionselective inhibitor of histone deacetylases. Proc Natl Acad Sci U S A 103: 1587-1592. CrossRef Medline

Slomnicki LP, Lésniak W (2008) A putative role of the amyloid precursor protein intracellular domain (AICD) in transcription. Acta Neurobiol Exp 68:219-228. Medline

Squatrito M, Gorrini C, Amati B (2006) Tip60 in DNA damage response and growth control: many tricks in one HAT. Trends Cell Biol 16:433442. CrossRef Medline

Stokin GB, Goldstein LS (2006) Axonal transport and Alzheimer's disease. Annu Rev Biochem 75:607-627. CrossRef Medline

Stokin GB Lillo C, Falzone TL, Brusch RG, Rockenstein E, Mount SL, Raman R, Davies P, Masliah E, Williams DS, Goldstein LS (2005) Axonopathy and transport deficits early in the pathogenesis of Alzheimer's disease. Science 307:1282-1288. CrossRef Medline
Sweatt JD (2010) Neuroscience. Epigenetics and cognitive aging. Science 328:701-702.

Torroja L, Chu H, Kotovsky I, White K (1999) Neuronal overexpression of APPL, the Drosophila homologue of the amyloid precursor protein (APP), disrupts axonal transport. Curr Biol 9:489-492. CrossRef Medline

Trinchese F, Fa' M, Liu S, Zhang H, Hidalgo A, Schmidt SD, Yamaguchi $\mathrm{H}$, Yoshii N, Mathews PM, Nixon RA, Arancio O (2008) Inhibition of calpains improves memory and synaptic transmission in a mouse model of Alzheimer disease. J Clin Invest 118:2796-2807. CrossRef Medline

Vecsey CG, Hawk JD, Lattal KM, Stein JM, Fabian SA, Attner MA, Cabrera SM, McDonough CB, Brindle PK, Abel T, Wood MA (2007) Histone deacetylase inhibitors enhance memory and synaptic plasticity via CREB: CBP-dependent transcriptional activation. J Neurosci 27:6128-6140. CrossRef Medline

Wang J, Weaver IC, Gauthier-Fisher A, Wang H, He L, Yeomans J, Wondisford F, Kaplan DR, Miller FD (2010) CBP histone acetyltransferase activity regulates embryonic neural differentiation in the normal and Rubinstein-Taybi syndrome brain. Dev Cell 18:114-125. CrossRef Medline

Zhang YQ, Rodesch CK, Broadie K (2002) Living synaptic vesicle marker: synaptotagmin-GFP. Genesis 34:142-145. CrossRef Medline

Zhu X, Singh N, Donnelly C, Boimel P, Elefant F (2007) The cloning and characterization of the histone acetyltransferase human homolog Dmel/ TIP60 in Drosophila melanogaster: Dmel/TIP60 is essential for multicellular development. Genetics 175:1229-1240. Medline 